\title{
Numerical approach for stochastic differential equations of fragmentation; application to avalanches
}

\author{
Lucian Beznea*, Madalina Deaconu ${ }^{\dagger}$ and Oana Lupaşcu ${ }^{\ddagger}$
}

\begin{abstract}
This paper builds and develops an unifying method for the construction of a continuous time fragmentation-branching processes on the space of all fragmentation sizes, induced either by continuous fragmentation kernels or by discontinuous ones. This construction leads to a stochastic model for the fragmentation phase of an avalanche. We introduce also an approximation scheme for the process which solves the corresponding stochastic differential equations of fragmentation. A new achievement of the paper is to compute the distributions of the branching processes approximating the forthcoming branching-fragmentation process. This numerical approach of the associated branching-fragmentation process, is, to our knowledge, one of the first in this direction. We present also numerical results that confirm the validity of the fractal property which was emphasized by our model for an avalanche.
\end{abstract}

AMS subject classification: 60J80, 65C30, 60K35, 82C80 60J45, 60J35.

Keywords: Fragmentation equation and kernel, avalanche, branching process and kernel, stochastic equation of fragmentation, numerical methods, space of fragmentation sizes, measure-valued process.

\section{Introduction}

Modeling avalanche formation of soils, snow, granular materials, or other geomaterials, is a complex, real-life three dimensional problem, possessing many challenges; see e.g. $[18,1]$. A deterministic numerical approach for the transport fragmentation-coagulation equation with power fragmentation rates and separable coagulation kernels is given in [16], by using a Laguerre pseudo-spectral method. In [9] it is presented a numerical method for an interacting one-dimensional avalanche process, by considering a related

* Simion Stoilow Institute of Mathematics of the Romanian Academy, Research unit No. 2, P.O. Box 1-764, RO-014700 Bucharest, Romania, University of Bucharest, Faculty of Mathematics and Computer Science, and Centre Francophone en Mathématique de Bucarest.

†Inria, Villers-lès-Nancy, F-54600, France; Université de Lorraine, CNRS, Institut Elie Cartan de Lorraine - UMR 7502, Vandoeuvre-lès-Nancy, F-54506, France.

${ }^{\ddagger}$ Gheorghe Mihoc-Caius Iacob Institute of Mathematical Statistics and Applied Mathematics of the Romanian Academy, Calea 13 Septembrie 13, RO-050711 Bucharest, Romania. Emails: lucian.beznea@imar.ro (L. Beznea), Madalina.Deaconu@inria.fr (M. Deaconu), oana.lupascu@yahoo.com (O. Lupaşcu) 
mean-field coagulation-fragmentation model. In the time discrete case, a probabilistic study of the Smoluchowski's coagulation equation and a numerical method are developed in $[11]$.

In this paper we give a probabilistic numerical approach for the time evolution of an avalanche-type process, by using a stochastic model for the fragmentation process of an infinite particles system, introduced previously by the authors in [4], and [5]. Let us also mention the study of coagulation-fragmentation models by probabilistic methods done in [10].

The key point of the model is to consider a particular fragmentation kernel related to a "rupture-type" property that occurs in the physical deterministic models for the avalanches, see for example [19]. More precisely, such a specific property means that the ratio between the dimensions of the resulting fragments after a particle splitting is constant in time.

A theoretical stochastic avalanche model involving these rupture properties was initiated in [5]. In particular, the continuous time evolution of the number of the resulting fragments is described by a specific branching Markov processes on the finite configurations of particles sizes. The main difficulty in simulating such an avalanche process is the discontinuity of the corresponding avalanche kernel. In order to overcome this problem, we consider a stochastic differential equation of fragmentation for avalanches associated to the first order integral operator induced by a discontinuous kernel. We present an algorithm to approximate the process, the weak solution of the specific stochastic equation. We also approximate the distribution of the branching process related to the fragmentation processes we investigate.

The paper is organized as follows.

In Section 2 we introduce the stochastic model for the pure fragmentation phenomenon with a continuous fragmentation kernel (see [10] and [4]), under hypotheses which ensure existence and uniqueness results. In Subsection 2.2 we present the case of a discontinuous bounded fragmentation kernel (cf. [5]), that we need further in our numerical scheme. In particular, we state and solve a stochastic differential equation of fragmentation associated to the integral operator induced by the discontinuous kernel for avalanches.

In Section 3 we briefly treat the related branching processes with state space the set of all finite configurations of the interval $[0,1]$. We remark the fractal property of such a branching-fragmentation process, in the case of the model for the avalanches, stated in assertion $(i v)$ of Corollary 6.

Section 4 is reserved to a probabilistic approach to simulate a fragmentation process and we give numerical results for some continuous and discontinuous fragmentation kernels. We also compute an exact solution of the fragmentation equation with constant fragmentation rate. Then we compare it with the approximation obtained by our algorithm and we observe that our approximation is very close to the exact solution. Subsection 4.2 is devoted to the probabilistic simulation of an avalanche-type process, the solution of the stochastic differential equation of fragmentation studied in Subsection 2.2. Finally, in Subsection 4.3 we state an algorithm approximating the distribution of the branching process occurring in our stochastic model of an avalanche. In particular, the numerical results illustrate the fractal property (from Section 3) we mentioned before.

The first two sections are based on results obtained in [4] and [5], where complements and related details may be found. 


\section{Stochastic differential equations of fragmentation}

We introduce in this section a binary fragmentation model. Consider an infinite system of particles, each particle being characterized by its mass. As time evolves the particles perform fragmentation, that is one particle can split into two smaller particles by conserving the total mass.

Let us introduce the fragmentation kernel $F$, that is, a symmetric function $F$ : $(0,1]^{2} \longrightarrow \mathbb{R}_{+}$. Recall that $F(x, y)$ represents the rate of fragmentation of a particle of size $x+y$ into two particles of sizes $x$ and $y$.

\subsection{Continuous fragmentation kernels}

The fragmentation equation describes the evolution of the concentration of particles of mass $x$ at time $t$. If we denote this quantity by $c(t, x)$ this equation writes:

$$
\left\{\begin{aligned}
\frac{\partial}{\partial t} c(t, x) & =\int_{x}^{1} F(x, y-x) c(t, y) \mathrm{d} y-\frac{1}{2} c(t, x) \int_{0}^{x} F(y, x-y) \mathrm{d} y \text { for all } t \geqslant 0 \text { and } x \in[0,1], \\
c(0, x) & =c_{0}(x) \text { for all } x \in[0,1] .
\end{aligned}\right.
$$

For a detailed description of the model see [10].

Assume that the fragmentation kernel $F:(0,1]^{2} \longrightarrow \mathbb{R}_{+}$is a continuous symmetric map and moreover, $F$ is supposed continuous from $[0,1]^{2}$ to $\mathbb{R}_{+} \cup\{+\infty\}$ and define the function $\psi:[0,1] \longrightarrow \mathbb{R}_{+}$as

$$
\psi(x):=\frac{1}{x} \int_{0}^{x} y(x-y) F(y, x-y) \mathrm{d} y \text { for } x>0 \text { and } \psi(0):=0,
$$

and we suppose that

$$
\psi \text { is a continuous function on }[0,1] \text {. }
$$

Recall that $\psi(x)$ represents the rate of loss of mass of particles of mass $x$.

With the notations from [10] consider the operator $\mathcal{F}$ defined as

$$
\mathcal{F} f(x)=\int_{0}^{x}[f(x-y)-f(x)] \frac{x-y}{x} F(y, x-y) \mathrm{d} y, x \in[0,1],
$$

where $\mathcal{F} f$ is well-defined for every Lipschitz continuous function $f$ on $E$, according to the above assumption (2.3) on $\psi$. We intend to write $\mathcal{F}$ as a first order integral operator and we present some preliminaries.

Let

$$
E:=[0,1]
$$

and $N$ be a kernel on $E, N \neq 0$. For all $x \in E$ we denote by $N_{x}$ the measure on $E$ induced by the kernel $N, N_{x}(A):=N\left(1_{A}\right)(x)$ for all $A \in \mathcal{B}(E)$. Suppose that $\int_{E}|x-y| N_{x}(\mathrm{~d} y)<\infty$ for all $x \in E$ and define the first order integral operator $\tilde{N}$ induced by $N$ as

$$
\tilde{N} f(x):=\int_{E}[f(y)-f(x)] N_{x}(\mathrm{~d} y) \quad \text { for all } x \in E,
$$


where $f$ belongs to the space of all Lipschitz continuous functions on $E$. If $N$ is a bounded kernel then $\widetilde{N}$ becomes a bounded linear operator on the space $b \mathcal{B}(E)$ of all bounded Borel measurable real-valued functions on $E$, and it is the generator of a (continuous time) jump Markov process.

Consider now the kernel $N^{F}$ on $E$, induced by $F$, defined as

$$
N^{F} f(x):=\int_{0}^{x} f(z) \frac{z}{x} F(x-z, z) \mathrm{d} z, \quad x \in E .
$$

The condition imposed to the function $\psi$ defined in (2.2) implies that $N^{F}$ satisfies the above mentioned integrability condition necessary to define the operator $\widetilde{N^{F}}$ and one can see immediately that $\mathcal{F}=\widetilde{N^{F}}$.

Let us define $g(t, x):=x c(t, x)$ for all $x \in[0,1]$. Under the conservation of mass assumption, that is $\int_{0}^{1} x c(t, x) \mathrm{d} x=1$, for all $t \in \mathbb{R}_{+}, g$ is a probability distribution function and we ca associate the stochastic differential equation of fragmentation associated with the kernel $F$ :

$$
X_{t}=X_{0}-\int_{0}^{t} \int_{0}^{1} \int_{0}^{1} y \mathbb{1}_{\left\{y \in\left(0, X_{s-}\right)\right\}} \mathbb{1}_{\left\{u \leqslant \frac{X_{S_{-}-y}}{X_{s^{-}}} F\left(y, X_{s-}-y\right)\right\}} p(\mathrm{~d} s, \mathrm{~d} y, \mathrm{~d} u), t \geqslant 0,
$$

where $p(\mathrm{~d} s, \mathrm{~d} y, \mathrm{~d} u)$ is an adapted Poisson measure on $[0,+\infty) \times(0,1] \times(0,1]$ with intensity measure $\mathrm{d} s \mathrm{~d} y \mathrm{~d} u$; see [10]. The relation between the fragmentation equation (2.1) and the stochastic differential equation of fragmentation (2.6) is that the density of $X_{t}$ is $g(t, \cdot)$.

The Fellerian case. We consider the fragmentation equation for the mass distribution:

$$
\left\langle Q_{t}, \phi\right\rangle=\left\langle Q_{0}, \phi\right\rangle+\int_{0}^{t}\left\langle Q_{s}, \mathcal{F} \phi\right\rangle \mathrm{d} s \text { for all } \phi \in \mathcal{C}^{1}(E) \text { and } t \geq 0
$$

where we denote $\left\langle Q_{t}, \phi\right\rangle=\int_{0}^{1} \phi(y) Q_{t}(\mathrm{~d} y)$ and for any $x \in E$ we define

$$
\mathcal{F} \phi(x)=\int_{0}^{x}[\phi(x-y)-\phi(x)] \frac{x-y}{x} F(y, x-y) \mathrm{d} y .
$$

Assume that for each $\varepsilon \in(0,1) \lim _{k \rightarrow+\infty} \sup _{x \geqslant \varepsilon} \frac{1}{x} \int_{0}^{x} y(x-y) F(y, x-y) \mathbb{1}_{\{F(y, x-y) \geqslant k\}} \mathrm{d} y=0$. Then for each probability $Q_{0}$ on $E$ there exists a solution $X$ of (2.6), the stochastic differential equation of fragmentation associated with the kernel $F$, with initial distribution $Q_{0}=\mathcal{L}\left(X_{0}\right)$. If we put $Q_{t}:=\mathcal{L}\left(X_{t}\right), t \geqslant 0$, then the family $\left(Q_{t}\right)_{t \geqslant 0}$ is a solution of $(2.7)$, the fragmentation equation for the mass distribution; see [10] and Proposition 2.1 from $[4]$.

For each $x \in E$ let $X_{x}=\left(X_{x, t}\right)_{t \geq 0}$ be the solution of the stochastic differential equation of fragmentation (2.6) associated with the kernel $F$ and the initial distribution $\delta_{x}$, i.e., $Q_{0}=\delta_{x}$.

Proposition 1. Suppose that for each $x \in E$, taking $Q_{0}=\delta_{x}$, the equation (2.7) has a unique solution $\left(Q_{t, x}\right)_{t \geq 0}$ and the function $E \ni x \longmapsto\left\langle Q_{t, x}, \phi\right\rangle$ is continuous for each $\phi \in \mathcal{C}^{1}(E)$ and $t>0$. Then the family of kernels $\left(Q_{t}\right)_{t \geq 0}$ on $[0,1]$, defined as

$$
Q_{t} f(x):=\left\langle Q_{t, x}, f\right\rangle, \quad f \in p b \mathcal{B}(E), x \in E,
$$


induces a Feller semigroup on $E$ and consequently it is the transition function of a standard (Markov) process $X^{0}=\left(\Omega, \mathcal{F}, \mathcal{F}_{t}, X_{t}^{0}, \mathbb{P}^{x}\right)$ with state space $E$; here $p b \mathcal{B}(E)$ denotes the positive, real-valued Borel measurable functions defined on $E$. In addition, for all $t \geq 0$ and $x \in E$, the solution $X_{x, t}$ of the stochastic differential equation of fragmentation (2.6) and $\left(X_{t}^{0}, \mathbb{P}^{x}\right)$ have the same distribution.

Proof. The semigroup property of $\left(Q_{t}\right)_{t \geqslant 0}$ is rather a straight-forward consequence of the uniqueness. Indeed, we have to show that $Q_{t^{\prime}+t} \phi=Q_{t}\left(Q_{t^{\prime}} \phi\right)$, so, it is enough to prove that the mapping $s \longmapsto Q_{t^{\prime}+s, x}$ verifies the equation (2.7) (with $Q_{0}=\delta_{x}, x \in E$ ) and $Q_{t^{\prime}} \phi$ instead of $\phi$. We have

$$
\begin{aligned}
\left\langle Q_{t^{\prime}+t, x} \phi\right\rangle & =\phi(x)+\int_{0}^{t^{\prime}}\left\langle Q_{s, x}, \mathcal{F} \phi\right\rangle \mathrm{d} s+\int_{0}^{t}\left\langle Q_{t^{\prime}+s, x}, \mathcal{F} \phi\right\rangle \mathrm{d} s \\
& =Q_{t^{\prime}} \phi(x)+\int_{0}^{t}\left\langle Q_{t^{\prime}+s, x}, \mathcal{F} \phi\right\rangle \mathrm{d} s .
\end{aligned}
$$

We claim that $\left(Q_{t}\right)_{t \geq 0}$ is a Feller semigroup on $E$. Indeed, if $\phi \in \mathcal{C}^{1}(E)$ then $\mathcal{F} \phi(x) \leq$ $\left\|\phi^{\prime}\right\|_{\infty} \psi(x), x \in E$, and by $(2.7)\left\|Q_{t} \phi-\phi\right\|_{\infty} \leq t\left\|\phi^{\prime}\right\|_{\infty}\|\psi\|_{\infty}$ for all $t>0$. The assertion follows by the density of $\mathcal{C}^{1}(E)$ in $\mathcal{C}(E)$. The existence of the standard process $X^{0}$ having $\left(Q_{t}\right)_{t \geq 0}$ as transition function is now a consequence of a main result on Feller processes, see e.g., [8], Theorem (9.4).

By Proposition 2.1 from [4], for each $x \in E$ we have $\mathcal{L}\left(X_{x, t}\right)=Q_{t, x}=\mathcal{L}\left(X_{t}^{0}\right)$.

We fix a sequence $\left(d_{n}\right)_{n \geqslant 1} \subset(0,1)$ of thresholds for the fragmentation dimensions, strictly decreasing to zero. For each $n \geqslant 1$ let $E_{n}:=\left[d_{n}, 1\right]$ and $E_{n}^{\prime}:=\left[d_{n+1}, d_{n}\right)$, $E_{0}^{\prime}:=E_{1}$.

As in Section 3 from [4], the main hypotheses are the following.

(H1) For each $n \geqslant 1$ there exists a conservative right Markov process $X^{n}$ with state space $E_{n}$ and transition function $\left(P_{t}^{n}\right)_{t \geqslant 0}$ such that $P_{t, x}^{n+1}=P_{t, x}^{n}$ for all $n \geqslant 1, t \geqslant 0$, and $x \in E_{n}$.

(H2) For every $n \geqslant 0$ the set $E_{n}^{\prime}$ is absorbing in $E_{n+1}$ with respect to the process $X^{n+1}$.

The hypotheses $(H 1)$ and $(H 2)$ are tailor-made for the application to the fragmentation processes. The compatibility between $P_{t}^{n+1}$ and $P_{t}^{n}$ stated in $(H 1)$ illustrates the fact that the Markov process $X^{n}$ is induced by a fragmentation in particles with "sizes" smaller than $d_{n}$, while the interpretation of condition (H2) is the following: if a particle is already smaller than $d_{n}$, then it cannot produce further "fragments" with bigger size than $d_{n}$. The hypotheses $(H 1)$ and (H2) imply that the set $E_{n}$ is also absorbing in $E_{n+1}$ with respect to the process $X^{n+1}$.

Truncated fragmentation kernels. Define for $n \geq 1$

$$
F_{n}(x, y):=\mathbb{1}_{\left(d_{n}, 1\right]}(x \wedge y) F(x, y), x, y \in E:=[0,1] .
$$

One can see that the kernel $N^{F^{n}}$ is bounded and therefore the operator $\widetilde{N^{F^{n}}}$ is the generator of a jump Markov process with state space $E$.

Proposition 2. If $n \geqslant 1$ then $E_{n}$ is an absorbing set with respect to the Markov process generated by $\widetilde{N^{F^{n}}}$ and the following assertions hold for the restriction $X^{n}=\left(X_{t}^{n}\right)_{t \geqslant 0}$ of this process to $E_{n}$. 
(i) If $t \geqslant 0$ and $x \in E_{n}$ then $P_{t}^{n}\left(\mathbb{1}_{(x, 1]}\right)(x)=0$ and the hypotheses (H1) and (H2) are satisfied.

(ii) Let $\mathrm{L}^{n}$ be the infinitesimal generator of $\left(P_{t}^{n}\right)_{t \geqslant 0}$, regarded as a $C_{0}$-semigroup of contractions on $b \mathcal{B}\left(E_{n}\right)$. Then for every $\phi \in b \mathcal{B}\left(E_{n}\right)$ we have $\mathrm{L}^{n} \phi=\sum_{k=1}^{n} \mathbb{1}_{E_{k-1}^{\prime}} \mathcal{F}_{k} \bar{\phi}$, where $\mathcal{F}_{k}$ is given by (2.8) with $F_{k}$ instead of $F$, and $\bar{\phi} \in b \mathcal{B}(E)$ is an extension of $\phi$ from $E_{n}$ to $E$. In particular, for each probability $\nu$ on $E_{n}$, the process $\phi\left(X_{t}^{n}\right)-\sum_{k=1}^{n} \int_{0}^{t}\left(\mathbb{1}_{E_{k-1}^{\prime}} \mathcal{F}_{k} \bar{\phi}\right)\left(X_{s}^{n}\right) \mathrm{d} s, t \geqslant 0$, is a martingale under $\mathbb{P}^{\nu}=\int \mathbb{P}^{x} \nu(\mathrm{d} x)$, with respect to the filtration of $X^{n}$.

(iii) For every $x \in E_{n}$ the stochastic differential equation of fragmentation associated with the kernel $F_{n}$ with the initial distribution $\delta_{x}$ has a solution which is equal in distribution with $\left(X^{n}, \mathbb{P}^{x}\right)$, where $p(\mathrm{~d} s, \mathrm{~d} y, \mathrm{~d} u)$ is an adapted Poisson measure on $[0,+\infty) \times(0,1] \times(0,1]$ with intensity measure $\mathrm{d} s \mathrm{~d} y \mathrm{~d} u$.

For the proof of the above proposition see [4], the proofs of Proposition 2.2 and Proposition 5.2 (for assertion $(i i)$ ). The martingale property from assertion $(i i)$ is a consequence of the Markov property of the process $X^{n}$.

\subsection{Discontinuous fragmentation kernels for avalanches}

In general, we cannot associate a stochastic equation of fragmentation like in assertion (iii) of Proposition 2 as the assumption that $F$ is continuous is not always valid. However, in Theorem 3 below it is written down and solved an appropriate stochastic equation of fragmentation, for the avalanche case, although the appropriate fragmentation kernel is discontinuous and we complete so results from [5]; for more details see Remark 4 below.

Consider again a fragmentation kernel $F:(0,1]^{2} \longrightarrow \mathbb{R}_{+}$. The following assumption is suggested by the so called rupture properties, emphasized in the deterministic modeling of the snow avalanches: 0 .

There exists a function $\Phi:(0, \infty) \longrightarrow(0, \infty)$ such that $F(x, y)=\Phi\left(\frac{x}{y}\right)$ for all $x, y>$

Since the fragmentation kernel $F$ is assumed to be a symmetric function, we have $\Phi(z)=\Phi\left(\frac{1}{z}\right)$ for all $z>0$. We give an example of such a fragmentation kernel as follows. Fix a "ratio" $r, 0<r<1$, and consider the fragmentation kernel $F^{r}:[0,1]^{2} \longrightarrow \mathbb{R}_{+}$, defined as $F^{r}(x, y):=\frac{1}{2}\left(\delta_{r}\left(\frac{x}{y}\right)+\delta_{1 / r}\left(\frac{x}{y}\right)\right)$, if $x, y>0$, and $F^{r}(x, y):=0$ if $x y=0$. We have $F^{r}(x, y)=\Phi^{r}\left(\frac{x}{y}\right)$ for all $x, y>0$, where $\Phi^{r}:(0, \infty) \longrightarrow(0, \infty)$ is defined as $\Phi^{r}(z):=\frac{1}{2}\left(\delta_{r}(z)+\delta_{1 / r}(z)\right), z>0$. Clearly, the function $\Phi^{r}$ is not continuous. By approximating the function $\Phi^{r}$ with a convenient sequence of continuous functions, one can see that the kernel $N^{F^{r}}$ associated with $F^{r}$ is given by the following linear combination of Dirac measures:

$$
N_{x}^{F^{r}}:=\lambda_{o}\left(\beta x \delta_{\beta x}+(1-\beta) x \delta_{(1-\beta) x}\right),
$$

where $\lambda_{o}:=\frac{\beta^{2}+(1-\beta)^{2}}{4}$ with $\beta:=\frac{r}{1+r}$. In this case the kernel $N^{F^{r}}$ is no more Markovian and has no density with respect to the Lebesgue measure. We assume that $d_{1}<\beta \leqslant 1 / 2$ and $d_{n+1} / d_{n}<\beta$ for all $n \geqslant 1$. Let $n \geqslant 1$ be fixed. Then $E_{n}=\bigcup_{k=1}^{n} E_{k-1}^{\prime}$. The kernel 
$N^{F^{r}}$ given by (2.9) is used to define the kernel $N_{n}^{r}$ on $E_{n}$ as

$$
N_{n}^{r} f:=\sum_{k=1}^{n} \mathbb{1}_{E_{k-1}^{\prime}} N^{F^{r}}\left(f \mathbb{1}_{E_{k-1}^{\prime}}\right) \text { for all } f \in p b \mathcal{B}\left(E_{n}\right) .
$$

Further, we consider the first order integral operator $\widetilde{N_{n}^{r}}$,

$$
\mathcal{F}_{n}^{r} f(x):=\widetilde{N_{n}^{r}} f(x)=\int_{E_{n}}[f(y)-f(x)]\left(N_{n}^{r}\right)_{x}(\mathrm{~d} y) \text { for all } f \in p b \mathcal{B}\left(E_{n}\right) \text { and } x \in E_{n} .
$$

The operator $\mathcal{F}_{n}^{r}$ is the generator of a (continuous time) jump Markov process $X^{r, n}=$ $\left(X_{t}^{r, n}\right)_{t \geqslant 0}$. Its transition function is $P_{t}^{r, n}:=e^{\mathcal{F}_{n}^{r} t}, t \geqslant 0$.

For every $x \in E$ let

$$
E_{\beta, x}:=\left\{\beta^{i}(1-\beta)^{j} x: i, j \in \mathbb{N}\right\} \cup\{0\} \text { and } E_{\beta, x, n}:=E_{\beta, x} \cap E_{n} \text { for } n \geqslant 1 .
$$

Theorem 3. If $n \geqslant 1$ then $E_{n}$ is an absorbing set with respect to the Markov process $X^{r, n}$ with state space $E_{n}$ and the following assertions hold.

(i) If $t \geqslant 0$ and $x \in E_{n}$ then $P_{t}^{r, n}\left(\mathbb{1}_{(x, 1]}\right)(x)=0$ and the hypotheses (H1) and (H2) are satisfied.

(ii) For every $\phi \in p b \mathcal{B}\left(E_{n}\right)$ and each probability $\nu$ on $E_{n}$, the process $\phi\left(X_{t}^{r, n}\right)-\int_{0}^{t} \mathcal{F}_{n}^{r} \phi\left(X_{s}^{r, n}\right) \mathrm{d} s$, $t \geqslant 0$, is a martingale under $\mathbb{P}^{\nu}$, with respect to the natural filtration of $X^{r, n}$.

(iii) If $x \in E_{n}, n \geqslant 1$, then the following stochastic differential equation of fragmentation for avalanches, with the initial distribution $\delta_{x}$, has a solution which is equal in distribution with $\left(X^{r, n}, \mathbb{P}^{x}\right)$ :

$$
\begin{aligned}
X_{t}=X_{0} & -\int_{0}^{t} \int_{0}^{\infty} p(\mathrm{~d} \alpha, \mathrm{d} s) X_{\alpha-} \sum_{k=1}^{n}\left((1-\beta) \mathbb{1}_{\left[\frac{d_{k}}{\beta} \leqslant X_{\alpha-}<d_{k-1}, \frac{s}{\lambda_{o} \beta}<X_{\alpha-}\right]}\right. \\
& \left.+\beta \mathbb{1}_{\left[\frac{d_{k}}{1-\beta} \leqslant X_{\alpha-}<\frac{d_{k}}{\beta}, \frac{s}{\lambda_{o}(1-\beta)}<X_{\alpha-} \leqslant \frac{s}{\lambda_{o} \beta}\right] \cup\left[\frac{d_{k}}{\beta} \leqslant X_{\alpha-}<d_{k-1}, \frac{s}{\lambda_{o}}<X_{\alpha-} \leqslant \frac{s}{\lambda_{o} \beta}\right]}\right),
\end{aligned}
$$

where $p(\mathrm{~d} \alpha, \mathrm{d} s)$ is a Poisson measure with intensity $q:=\mathrm{d} \alpha \mathrm{d} s$.

(iv) If $x \in E_{n}$ then $\mathbb{P}^{x}$-a.s. $X_{t}^{r, n} \in E_{\beta, x, n}$ for all $t \geqslant 0$.

Proof. For the proof of the assertions (i), (ii), and (iv) we refer to Proposition 2.2 and Remark 2.1 (ii) from [5].

(iii) We argue as in the proof of Theorem 3.1 from [5]. Define the bounded kernel $K^{n}$ on $\mathbb{R}$ by $K_{x}^{n}:=\lambda_{o} x\left[\beta \mathbb{1}_{E_{k-1}^{\prime}}(\beta x) \delta_{(\beta-1) x}+(1-\beta) \mathbb{1}_{E_{k-1}^{\prime}}((1-\beta) x) \delta_{-\beta x}\right]$ if $x \in E_{k-1}^{\prime}$, $1 \leqslant k \leqslant n$, and $K_{x}^{n}:=0$ else. One can see that $\mathcal{F}_{n}^{r} f(x)=\int_{\mathbb{R}}[f(x+y)-f(x)] K_{x}^{n}(\mathrm{~d} y)$ for all $f \in p b \mathcal{B}(\mathbb{R})$ and $x \in \mathbb{R}$, where on the left hand side the kernel $N_{n}^{r}$ occurring in the definition of $\mathcal{F}_{n}^{r}$ is extended from $E_{n}$ to $\mathbb{R}$ with zero on the complement of $E_{n}$. In particular, we have $K^{n} f(x)=\int f(y-x)\left(N_{n}^{r}\right)_{x}(\mathrm{~d} y)$ for $x \in E_{n}$. By Lemma (2.2) 
from [8] applied to the measure $\left(N_{n}^{r}\right)_{x}$ which is carried by $(0, x)$, we get for all $x, s \in \mathbb{R}$ : $K^{n} f(x)=\int_{\mathbb{R}} f\left(\tau^{n}(x, s)\right) \mathrm{d} s$, where $\tau^{n}(x, s):=\inf \left\{v>0:\left(N_{n}^{r}\right)_{x}((0, v])>s\right\}-x$ if $0 \leqslant s, x \in E_{n}$, and $\tau^{n}(x, s):=0$ else with the convention $f(\infty)=0$. Consider the stochastic differential equation from [13], page 479:

$$
\mathrm{d} X_{t}=b^{n}\left(X_{t-}\right) \mathrm{d} t+w_{t}^{n} \mathrm{~d} p_{t}-w_{t}^{n} \mathbb{1}_{\left[\left|w_{t}^{n}\right| \leqslant 1\right]} \mathrm{d} q_{t},
$$

where

$b^{n}(x):=2 \lambda_{o} \beta(\beta-1) x^{2}\left(\mathbb{1}_{E_{k-1}^{\prime}}(\beta x)+\mathbb{1}_{E_{k-1}^{\prime}}((1-\beta) x)\right)$ if $x \in E_{k-1}^{\prime}, 1 \leqslant k \leqslant n$, and $b^{n}(x):=0$ elsewhere, and where $w_{t}^{n}$ is a process that depends on $X_{t}$, such that

$$
K^{n} f\left(X_{t-}\right)=\int_{\mathbb{R}} \mathbb{1}_{\left[w_{t}^{n}(\cdot, s) \neq 0\right]} f\left(w_{t}^{n}(\cdot, s)\right) \mathrm{d} s .
$$

The existence of the process $w_{t}^{n}$ follows by Theorem (14.53) from [13], applied to the measure $\mathrm{d} s$. Here we can take $w_{t}^{n}(\cdot, s)=\tau^{n}\left(X_{t-}, s\right)$, hence

$$
\begin{aligned}
w_{t}^{n}(\cdot, s)= & \mathbb{1}_{[0, \infty)}(s) \sum_{k=1}^{n}(\beta-1) X_{t-} \mathbb{1}_{\left[\frac{d_{k}}{\beta} \leqslant X_{t-}<d_{k-1}, \frac{s}{\lambda_{o} \beta}<X_{t-}\right]} \\
& -\beta X_{t-} \mathbb{1}_{\left[\frac{d_{k}}{1-\beta} \leqslant X_{t-}<\frac{d_{k}}{\beta}, \frac{s}{\lambda_{o}(1-\beta)}<X_{t-} \leqslant \frac{s}{\lambda_{o} \beta}\right] \cup\left[\frac{d_{k}}{\beta} \leqslant X_{t-}<d_{k-1}, \frac{s}{\lambda_{o}}<X_{t-} \leqslant \frac{s}{\lambda_{o} \beta}\right]} \\
& +\infty\left(\mathbb{1}_{\left[X_{t-}<\frac{d_{k}}{1-\beta}\right] \cup\left[X_{t-} \leqslant \frac{s}{\lambda_{o}}\right]}+\mathbb{1}_{\left[\frac{d_{k}}{1-\beta} \leqslant X_{t-}<\frac{d_{k}}{\beta}\right]} \mathbb{1}_{\left[X_{t-} \leqslant \frac{s}{\lambda_{o}(1-\beta)}\right]} .\right.
\end{aligned}
$$

On the other hand observe that

$$
b^{n}(x)=\int_{\mathbb{R}} y K_{x}^{n}(\mathrm{~d} y)=\int_{0}^{\infty} \tau^{n}(x, s) \mathrm{d} s, x \in \mathbb{R} .
$$

Consequently, the stochastic differential equation considered by Jacod takes the form claimed in assertion $(i i i)$. Let further $\left(\mathcal{K}^{n}, C_{b}^{1}(\mathbb{R})\right)$ be the operator defined as:

$$
\mathcal{K}^{n} f(x)=b^{n}(x) f^{\prime}(x)+\int_{\mathbb{R}}\left[f(x+y)-f(x)-y \mathbb{1}_{\{|y| \leq 1\}} f^{\prime}(x)\right] K_{x}^{n}(\mathrm{~d} y), x \in \mathbb{R} ;
$$

cf. [13], page 434. From the above considerations we have $\mathcal{F}_{n}^{r}=\mathcal{K}^{n}$. Since by assertion (ii) we know that the martingale problem associated with the bounded operator $\mathcal{F}_{n}^{r}$ has a solution, Theorem (14.80) from [13], page 481, implies the existence of the solution of the stochastic differential equation (2.10).

Remark 4. A simplified version of (2.10), the stochastic differential equation of fragmentation for avalanches, was stated and solved in [5], the equation (3.1) and Theorem 3.1 respectively. Essentially, the completion in Theorem 3 is to take into account the sequence of thresholds for the fragmentation dimensions $\left(d_{n}\right)_{n \geqslant 1}$.

\section{Branching-fragmentation processes}

\subsection{Branching processes on the space of all fragmentation sizes}

For a Borel subset $A$ of $[0,1]$ define the space of finite configurations of $A$, which is the following set $\widehat{A}$ of finite positive measures on $A$ : 


$$
\widehat{A}:=\left\{\sum_{k \leqslant k_{0}} \delta_{x_{k}}: k_{0} \in \mathbb{N}^{*}, x_{k} \in A \text { for all } 1 \leqslant k \leqslant k_{0}\right\} \cup\{\mathbf{0}\},
$$

where $\mathbf{0}$ denotes the zero measure.

We identify $\widehat{A}$ with the union of all symmetric $m$-th powers $A^{(m)}$ of $A$ : $\widehat{A}=\bigcup_{m \geqslant 0} A^{(m)}$, where $A^{(0)}:=\{\mathbf{0}\}$; see, e.g., [12], [7], [6], and [17]. The set $\widehat{A}$ is endowed with the topology of disjoint union of topological spaces and the corresponding Borel $\sigma$-algebra $\mathcal{B}(\widehat{A})$.

If $p_{1}, p_{2}$ are two finite measures on $\widehat{A}$, then their convolution $p_{1} * p_{2}$ is the finite measure on $\widehat{A}$ defined for every $h \in p \mathcal{B}(\widehat{A})$ by

$$
\int_{\widehat{A}} p_{1} * p_{2}(\mathrm{~d} \nu) h(\nu):=\int_{\widehat{A}} p_{1}\left(\mathrm{~d} \nu_{1}\right) \int_{\widehat{A}} p_{2}\left(\mathrm{~d} \nu_{2}\right) h\left(\nu_{1}+\nu_{2}\right) .
$$

If $\varphi \in p \mathcal{B}(A)$, define the multiplicative function $\widehat{\varphi}: \widehat{A} \longrightarrow \mathbb{R}_{+}$as

$$
\widehat{\varphi}(\mathbf{x}):=\prod_{k} \varphi\left(x_{k}\right), \text { if } \mathbf{x}=\left(x_{k}\right)_{k \geqslant 1} \in \widehat{A}, \mathbf{x} \neq \mathbf{0}, \text { and } \widehat{\varphi}(\mathbf{0}):=1 .
$$

Recall that a bounded kernel $N$ on $\widehat{A}$ is called branching kernel if $N_{\mu+\nu}=N_{\mu} *$ $N_{\nu}$ for all $\mu, \nu \in \widehat{A}$, where $N_{\mu}$ denotes the measure on $\widehat{E}$ such that $\int g \mathrm{~d} N_{\mu}=N g(\mu)$, for all $g \in p \mathcal{B}(\widehat{A})$. Note that if $N$ is a branching kernel on $\widehat{A}$ then $N_{\mathbf{0}}=\delta_{\mathbf{0}} \in M(\widehat{E})$.

A right (Markov) process with state space $\widehat{A}$ is called branching process provided that its transition function is formed by branching kernels. The probabilistic description of a branching process is the following: if we take two independent versions $X$ and $X^{\prime}$ of the process, starting respectively from two measures $\mu$ and $\mu^{\prime}$, then $X+X^{\prime}$ and the process starting from $\mu+\mu^{\prime}$ are equal in distribution.

Suppose that for all $n \geqslant 1, B^{n}$ is a given Markovian kernel from $\widehat{E_{n}}$ to $E_{n}$ such that $(H 3)$

$$
\sup _{x \in E_{n}} B^{n} l_{1}(x)<\infty \text { and } B_{x}^{n+1}=B_{x}^{n} \text { for all } x \in E_{n}, n \geqslant 1,
$$

where for a function $f \in p \mathcal{B}\left(E_{n}\right)$ we consider the mapping $l_{f}: \widehat{E_{n}} \longrightarrow \mathbb{R}_{+}$defined as $l_{f}(\mu):=\int f \mathrm{~d} \mu, \mu \in \widehat{E_{n}}$. The kernel $B^{n}$ will control the non-local branching of a forthcoming process with state space the finite configurations of $E_{n}$. The first step is to apply Proposition 4.1 from [6], to construct a transition function of branching kernels $\left(\widehat{P_{t}^{n}}\right)_{t \geqslant 0}$ on $\widehat{E_{n}}$, induced by $\left(P_{t}^{n}\right)_{t \geqslant 0}$ and by the kernel $B^{n}$.

As in [4], we intend to construct a process with state space the set $S^{\downarrow}$ of all decreasing numerical sequences bounded above from 1 and with limit 0 ,

$$
S^{\downarrow}:=\left\{\mathbf{x}=\left(x_{k}\right)_{k \geqslant 1} \subseteq[0,1]:\left(x_{k}\right)_{k \geqslant 1} \text { decreasing, } \lim _{k} x_{k}=0\right\} .
$$

Recall that a sequence $\mathbf{x}$ from $S^{\downarrow}$ may be considered as "the sizes of the fragments resulting from the split of some block with unit size" (cf. [2], page 16). It is convenient to identify a sequence $\mathbf{x}=\left(x_{k}\right)_{k \geqslant 1}$ from $S^{\downarrow}$ with the $\sigma$-finite measure $\mu_{\mathbf{x}}$ on $[0,1]$, defined as $\mu_{\mathbf{x}}:=\sum_{k} \delta_{x_{k}}$ if $\mathbf{x} \neq \mathbf{0}$ and $\mu_{\mathbf{0}}=\mathbf{0}$, where the zero constant sequence $\mathbf{0}$ is identified with the zero measure, $\mu_{\mathbf{0}}=\mathbf{0}$. For $\mathbf{x} \in S^{\downarrow}$ we write $\mathbf{x}=\mu_{\mathbf{x}}$ where it is necessary to emphasize the identification of the sequence $\mathbf{x}$ with the measure $\mu_{\mathbf{x}}$.

In order to consider branching kernels on $S^{\downarrow}$ we need to have a convolution operation between finite measures on $S^{\downarrow}$. We first endow $S^{\downarrow}$ with a semigroup structure: if $\mathbf{x}, \mathbf{y} \in S^{\downarrow}$ then the sequence $\mathbf{x}+\mathbf{y} \in S^{\downarrow}$ is by definition the decreasing rearrangement of the terms of the sequences $\mathbf{x}$ and $\mathbf{y}$. The convolution may be now introduced as in the case of the 
space of all finite configurations, cf. (3.1): if $p_{1}, p_{2}$ are two finite measures on $S^{\downarrow}$, then their convolution $p_{1} * p_{2}$ is the finite measure on $S^{\downarrow}$, defined for every $h \in p \mathcal{B}\left(S^{\downarrow}\right)$ by:

$$
\int_{S \downarrow} p_{1} * p_{2}(\mathrm{~d} \nu) h(\nu):=\int_{S \downarrow} p_{1}\left(\mathrm{~d} \nu_{1}\right) \int_{S^{\downarrow}} p_{2}\left(\mathrm{~d} \nu_{2}\right) h\left(\nu_{1}+\nu_{2}\right) .
$$

The branching kernels on $S^{\downarrow}$ and the branching process with state space $S^{\downarrow}$ are now defined analogously, and the probabilistic interpretation remains valid. Define the mapping $\alpha_{n}: S^{\downarrow} \longmapsto \widehat{E_{n}}$ as $\alpha_{n}(\mathbf{x}):=\left.\mu_{\mathbf{x}}\right|_{E_{n}}, \mathbf{x}=\mu_{\mathbf{x}} \in S^{\downarrow}$. We have $\alpha_{n}(\mathbf{0})=\mathbf{0}$ and $\left.\alpha_{n}\right|_{\widehat{E_{n}}}=\operatorname{Id}_{\widehat{E_{n}}}$. Below, the notation $\mathbf{y} \leqslant \mathbf{x}$ for $\mathbf{x} \in \widehat{E}$ and $\mathbf{y} \in S^{\downarrow}$, means that $y_{k} \leqslant x_{j}$ for all $k \geqslant 1$ and some index $j$.

The next theorem extends essentially the main results from [4] and [5] on the construction of branching processes on the space of all finite configurations; see Corollary 6 below for particular applications.

Theorem 5. Assume that $(H 1),(H 2)$, and $(H 3)$ are in force. Then the following assertions hold.

(i) There exists a branching semigroup $\left(\widehat{P}_{t}\right)_{t \geqslant 0}$ on $S^{\downarrow}$, obtained as the projective limit of the sequence $\left(\widehat{P_{t}^{n}}\right)_{n \geqslant 1}$, i.e., for every $\mathbf{x} \in S^{\downarrow}, \mathbf{x}_{n}:=\alpha_{n}(\mathbf{x}) \in \widehat{E_{n}}, n \geqslant 1$, and $t>0$ the sequence of probability measures $\left(\widehat{P_{t, \mathbf{x}_{n}}^{n}}\right)_{n \geqslant 1}$ is projective with respect to $\left(\widehat{E_{n}}, \alpha_{n}\right)_{n \geqslant 1}$ and its limit is $\widehat{P_{t, \mathbf{x}}}$, that is $\widehat{P_{t, \mathbf{x}_{n+1}}^{n+1}} \circ \alpha_{n}^{-1}=\widehat{P_{t, \mathbf{x}_{n}}^{n}}$ and $\widehat{P_{t, \mathbf{x}}} \circ \alpha_{n}^{-1}=\widehat{P_{t, \mathbf{x}_{n}}^{n}}$ for all $n \geqslant 1$.

(ii) Suppose in addition that for every $n \geqslant 1\left(\widehat{P_{t}^{n}}\right)_{t \geqslant 0}$ is the transition function of a standard Markov process with state space $\widehat{E_{n}}$ and $P_{t}^{n}\left(\mathbb{1}_{(x, 1]}\right)(x)=0$ for all $t \geqslant 0$ and $x \in E_{n}$. Then $\left(\widehat{P}_{t}\right)_{t \geqslant 0}$ is the transition function of a branching process $\widehat{X}=\left(\widehat{X}_{t}\right)_{t \geqslant 0}$ with state $S^{\downarrow}$, and the following fragmentation property holds: if $\mathbf{x} \in \widehat{E}$ and $\mathbf{y} \in S^{\downarrow}$, $\mathbf{y} \leqslant \mathbf{x}$, then $\mathbb{P}^{\mathbf{y}}-$ a.s. $\widehat{X}_{t} \leqslant \mathbf{x}$.

\section{Sketch of the proof.}

(i) By Proposition 4.6 from [4], using the hypothesis (H3), we deduce that for each $t \geqslant 0$ the sequence of probability measures $\left(\widehat{P_{t, \mathbf{x}_{n}}^{n}}\right)_{n \geqslant 1}$ is projective as it was claimed. Let $S_{\infty}:=\left\{\left(\mathbf{x}^{n}\right)_{n \geqslant 1} \in \prod_{n \geqslant 1} \widehat{E_{n}}: \mathbf{x}^{n}=\alpha_{n}\left(\mathbf{x}^{m}\right)\right.$ or all $\left.m>n \geqslant 1\right\}$. By Proposition 4.5 from [4] the mapping $i: S^{\downarrow} \longrightarrow S_{\infty}$, defined as $i(\mathbf{x}):=\left(\alpha_{n}(\mathbf{x})\right)_{n \geqslant 1}, \mathbf{x} \in S^{\downarrow}$, is a bijection. Using this identification of $S^{\downarrow}$ with $S_{\infty}$ (equipped with the product topology), the above projective system of probabilities may be used to apply Bochner-Kolmogorov Theorem (see, e.g., [3]) in order to obtain the transition function $\left(\widehat{P}_{t}\right)_{t \geqslant 0}$ on $S^{\downarrow}$, as in Proposition 4.7 from [4].

To show that each kernel $\widehat{P}_{t}, t \geqslant 0$, is a branching kernel on $S^{\downarrow}$, we can proceed as in the particular case from [5], the proof of assertion $(i)$ of Theorem 4.3. For the reader convenience we write down here the main arguments. We have to check that $\widehat{P_{t, \mathbf{x}+\mathbf{y}}}(g)=\widehat{P_{t, \mathbf{x}}} * \widehat{P_{t, \mathbf{y}}}(g)$ for all $\mathbf{x}, \mathbf{y} \in S^{\downarrow}$, and $g \in p b \mathcal{B}\left(S^{\downarrow}\right)$. By a monotone class argument and using (5.10) from [4], we may assume that $g=f \circ \alpha_{n}$ for some $n \geqslant 1$, with $f \in p b \mathcal{B}\left(\widehat{E_{n}}\right)$. It remains to prove that $\widehat{P_{t, \mathbf{x}_{n}+\mathbf{y}_{n}}^{n}}(f)=\widehat{P_{t, \mathbf{x}_{n}}^{n}} * \widehat{P_{t, \mathbf{y}_{n}}^{n}}(f)$ for all $f \in p b \mathcal{B}\left(\widehat{E_{n}}\right)$, however, this equality is precisely the branching property of the kernel $\widehat{P_{t}^{n}}$ on $E_{n}$, which 
clearly holds.

(ii) The existence of the process $\widehat{X}$ with the transition function $\left(\widehat{P}_{t}\right)_{t \geqslant 0}$ is a consequence of Theorem 5.3 from [4]. By assertion $(i)$ it follows that $\widehat{X}$ is a branching process with state $S^{\downarrow}$. To prove the fragmentation property of $\widehat{X}$, that is the last part of assertion (ii), we may argue as in the proof of Corollary 5.5 from [4].

\subsection{Construction of branching-fragmentation processes; appli- cation to avalanches}

If $d>0$ and $g \in p b \mathcal{B}([d, 1])$ we consider the function ${ }_{d} g \in p b \mathcal{B}([0,1])$, the extension of $g$ to $[0,1]$ with the value $g(d)$ on $[0, d)$,

$$
{ }_{d} g(y):=g(d) \mathbb{1}_{[0, d)}(y)+g(y) \mathbb{1}_{[d, 1]}(y), y \in[0,1] .
$$

Define further the kernel $B_{d}: p \mathcal{B}(\widehat{[d, 1]}) \longrightarrow p \mathcal{B}([d, 1])$ as

$$
B_{d} h(x):=\frac{6}{x^{3}} \int_{0}^{x} y(x-y)_{d}\left(h^{(2)}\right)(y) \mathrm{d} y, x \in[d, 1],
$$

where for every $h \in p b \mathcal{B}(\widehat{[d, 1]})$ the function $h^{(2)} \in p b \mathcal{B}([d, 1])$ is defined as

$$
h^{(2)}(y):=\left.h\right|_{[d, 1]}(2)\left(2 \delta_{y}\right) \text { for all } y \in[d, 1],
$$

with $[d, 1]^{(2)}=\left\{\delta_{x_{1}}+\delta_{x_{2}}: x_{1}, x_{2} \in[d, 1]\right\}$. The kernel $B_{d}$ is Markovian and for each $x \geqslant d$ the probability measure $B_{d, x}$ is carried by $[d, 1]^{(2)}$; recall that $B_{d, x}$ is the probability measure induced by the Markovian kernel $B_{d}, B_{d, x}(A):=B_{d}\left(\mathbb{1}_{A}\right)(x)$ for all $A \in \mathcal{B}([d, 1])$.

Let $n \geqslant 1$ and consider the kernel $B^{n}$ from $\widehat{E_{n}}$ to $E_{n}$ defined as

$$
B^{n} h:=\sum_{k=1}^{n} \mathbb{1}_{E_{k-1}^{\prime}} B_{d_{k}} h, h \in p b \mathcal{B}\left(\widehat{E_{n}}\right) .
$$

In particular, $B^{1}=B_{d_{1}}$, for each $n \geqslant 1$ the kernel $B^{n}$ is Markovian, and the probability measure $B_{x}^{n}, x \in E_{n}$, is carried by the diagonal of $E_{n}^{(2)}=\left\{\delta_{x_{1}}+\delta_{x_{2}}: x_{1}, x_{2} \in E_{n}\right\}$; see [4] for further properties of the kernel $B^{n}$, related to fragmentation.

For the announced application to the avalanche model, we consider some specific branching mechanisms, cf. [5]. Fix a rupture factor $r \in(0,1)$, corresponding to an uniform proportionality of the fragments, which remains constant in time. For all $n \geqslant 1$ define the Markovian kernel $B^{r, n}$ from $\widehat{E_{n}}$ to $E_{n}$ as

$$
B^{r, n} h(x):=\frac{1}{a(x)} \sum_{1 \leqslant k \leqslant n} \sum_{E_{\beta, x} \ni y \leq x} \mathbb{1}_{E_{k-1}^{\prime}}(x)_{d_{k}} h(y, y) y(x-y), h \in p b \mathcal{B}\left(\widehat{E_{n}}\right), x \in E_{n},
$$

where $a(x):=\sum_{E_{\beta, x} \ni y \leq x} y(x-y)<\infty$ for all $x \in E_{n}$.

If $x_{1}, \ldots, x_{k} \in E$ and $\mathbf{x}=\delta_{x_{1}}+\ldots+\delta_{x_{k}} \in \widehat{E}$, we put $E_{\beta, \mathbf{x}}:=\bigcup_{j=1}^{k} E_{\beta, x_{j}}$ and $E_{\beta, \mathbf{x}, n}:=\bigcup_{j=1}^{k} E_{\beta, x_{j}, n}$.

Corollary 6. Let $n \geqslant 1$. Then the following assertions hold. 
(i) There exists a branching standard process with state space $\widehat{E_{n}}$, induced by the transition function $\left(P_{t}^{n}\right)_{t \geqslant 0}$ and by the kernel $B^{n}$.

(ii) There exists a branching standard process $\widehat{X^{r, n}}=\left(\widehat{X_{t}^{r, n}}\right)_{t \geqslant 0}$ with state space $\widehat{E_{n}}$, induced by the transition function $\left(P_{t}^{r, n}\right)_{t \geqslant 0}$ and by the kernel $B^{r, n}$. For every $\mathbf{x} \in \widehat{E_{n}}$, $\mathbf{y} \in \widehat{E_{\beta, \mathbf{x}, n}}$, and $t \geqslant 0$ we have $\mathbb{P}^{\mathbf{y}}-$ a.s. $\widehat{X_{t}^{r, n}} \in \widehat{E_{\beta, \mathbf{x}, n}}$.

(iii) There exists a branching right (Markov) process with state space $S^{\downarrow}$, having càdlàg trajectories, as stated in Theorem 5, associated with a continuous fragmentation kernel F.

(iv) There exists a branching right process $\widehat{X^{r}}=\left(\widehat{X_{t}^{r}}\right)_{t \geqslant 0}$ with state space $S^{\downarrow}$, having càdlàg trajectories, as stated in Theorem 5, associated with the discontinuous fragmentation kernel $F^{r}$ for avalanches. For each $\mathbf{x} \in \widehat{E}$, the set $S_{\beta, \mathbf{x}}^{\downarrow}:=\left\{\mathbf{y}=\left(y_{k}\right)_{k \geqslant 1} \in\right.$ $S^{\downarrow}: y_{k} \in E_{\beta, \mathbf{x}}$ for all $\left.k \geqslant 1\right\}$ is absorbing in $S^{\downarrow}$, that is, if $\mathbf{y} \in S_{\beta, \mathbf{x}}^{\downarrow}$ then $\mathbb{P}_{-a . s .}$ $\widehat{X_{t}^{r}} \in S_{\beta, \mathbf{x}}^{\downarrow}$ for all $t \geqslant 0$.

Proof. Observe first that both kernels $B^{n}$ and $B^{r, n}$ satisfy hypothesis (H3). The assertions follow applying Theorem 5, using Proposition 2 and Theorem 3.

Assertion ( $i v)$ of Corollary 6 gives a stochastic model for the fragmentation phase of an avalanche. The last part of assertion $(i v)$ emphasizes a fractal property of an avalanche, closed to its real physical properties: if we regard the fragmentation-branching process $\widehat{X^{r}}$ on the set $S_{\beta, \mathbf{x}}^{\downarrow}$ (which is possible by restriction, since $S_{\beta, \mathbf{x}}^{\downarrow}$ is absorbing according to assertion ( $i v$ ) of Corollary 6), then independently with respect to the sequence of sizes $\mathbf{x}$ of the initial fragments, from the moment when the avalanche started, and remaining constant in time, the ratio between the resulting fragments are all powers of $\beta$.

\section{Simulation for fragmentation processes}

\subsection{Continuous fragmentation kernel}

In this section we present numerical results obtained by approximating the process which is the weak solution of the stochastic differential equation of fragmentation in the case of a continuous fragmentation kernel. It is precisely the equation (2.6) from assertion (iii) of Proposition 2, with $F$ instead of $F_{n}$. We test also our results on a particular fragmentation kernel, for which an explicit solution exists.

Recall that, according to the probabilistic interpretation for the solution of the stochastic differential equation of fragmentation (2.6), the fragmentations occur at some Poisson random instants smaller than a final time $T$. Below we present the numerical algorithm. We denote by $\mathcal{U}([a, b])$ the uniform law on the interval $[a, b]$, for $a<b$, and by $\operatorname{Exp}(\lambda)$ the exponential law of parameter $\lambda$.

\section{Algorithm}

Initialization: Sample the initial particle $X_{0} \sim Q_{0}$. Set $T_{0}=0$. 
Step p: Sample a random variable $y \sim \mathcal{U}\left(\left[0, X_{p-1}\right]\right)$.

Compute $m_{p}=\frac{X_{p-1}-y}{X_{p-1}} F\left(y, X_{p-1}-y\right)$.

Sample a random variable $S_{p} \sim \operatorname{Exp}\left(m_{p}\right)$.

Set $T_{p}=T_{p-1}+S_{p}$.

Sample a random variable $u \sim \mathcal{U}([0,1])$.

If $u \leq m_{p}$, then a fragmentation occurs and set $X_{p}=X_{p-1}-y$.

Else set $X_{p}=X_{p-1}$.

Stop: When $T_{p}>T$.

Outcome: The approximated particle mass at $T, X_{p-1}$.

Numerical results. In order to perform numerical calculus we consider the following context. First, we take the size of the initial particle to be $Q_{0} \sim \mathcal{U}([0,1])$ and we also fix the final time $T$. We consider the following fragmentation rates: $F(x, y)=$ $x+y, F(x, y)=2, F(x, y)=\frac{1}{x+y+1}$, and $F(x, y)=\frac{2}{(x+y)^{3}}$ with the assumption $x \cdot y \neq 0$.

In Figure 1 we illustrate the paths of the processes corresponding to the above mentioned fragmentation rates for $T=50$.

We can note that the path of the stochastic process strongly depends on the form of the fragmentation kernel. For example for the kernel $F(x, y)=2 /(x+y)^{3}$ the paths is rapidly decreasing to 0 , this means that the initial particle splits quickly and the time between successive fragmentations is very short. The size of the fragment is thus close to zero after a small time. Among the four kernels this one is the larger one when $x+y$ is close to 0 and this contributes to define the fragmentation time and the size of the fragments.

\begin{tabular}{|c|c|c|}
\hline$F(x, y)$ & Mean $\widehat{I}_{M}$ & Confidence interval size (half) \\
\hline$x+y$ & 0.3609 & 0.0044 \\
\hline 2 & 0.0319 & 0.0012 \\
\hline$\frac{1}{x+y+1}$ & 0.4759 & 0.0062 \\
\hline$\frac{2}{(x+y)^{3}}$ & 0.0167 & 0.0013 \\
\hline
\end{tabular}

Table 1: Monte Carlo estimators for $M=10^{4}$ simulations, with $Q_{0} \sim \mathcal{U}([0,1])$ and $T=50$.

We give now an unbiased estimator of the mean of these random variables $\mathbb{E}\left(X_{T}\right)$, by using Monte Carlo method. In Table 1 it is written the Monte Carlo estimator of $\mathbb{E}\left(X_{T}\right)$, i.e., $\widehat{I}_{M}=\frac{1}{M} \sum_{i=1}^{M} X_{T}^{i}$ for each continuous fragmentation rate considered above. We consider here again $T=50, Q_{0} \sim \mathcal{U}([0,1])$, and the Monte Carlo parameter is $10^{4}$.

The variance of this estimator is given by $\hat{V}_{M} / M$ where $\hat{V}_{M}$ is the estimator of the variance $\operatorname{Var}\left(X_{T}\right)$. We report in the Table 1 also the confidence interval size.

The mean by Euler and Monte-Carlo. We represent in Figure 2 and Figure 3 the evolution in time of $\mathbb{E}\left(X_{t}\right)$ for $t \in[0,50]$. We observe that this function is decreasing and 

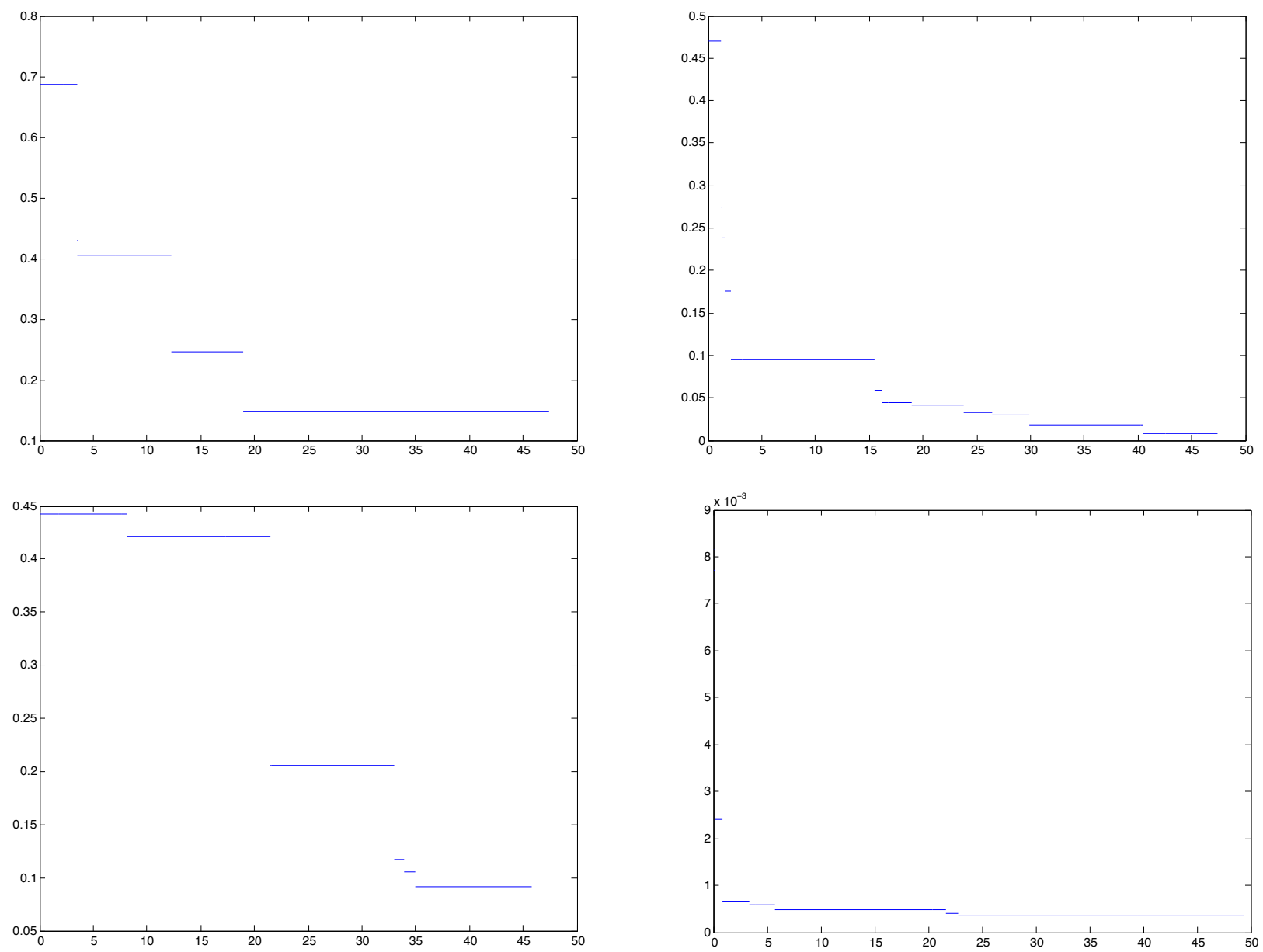

Figure 1: The paths of the fragmentation process for $F(x, y)=x+y, F(x, y)=$ $2, F(x, y)=1 /(x+y+1), F(x, y)=2 /(x+y)^{3}$, with $Q_{0} \sim \mathcal{U}([0,1])$ and $T=50$.

its form depends on the fragmentation kernel with similar behaviors as those observed for the Table 1.

\section{Comparison with the exact solution of the fragmentation equa- tion}

We consider the fragmentation kernel $F(x, y)=2$ for $x, y \in[0, \infty)$. In this case the fragmentation equation (2.1) writes

$$
\left\{\begin{array}{llll}
\frac{\partial}{\partial t} c(t, x) & =2 \int_{x}^{\infty} c(t, y) \mathrm{d} y-x c(t, x) & & \text { for all } x \geqslant 0 \\
c(0, x) & =c_{0}(x) & & \text { for all } x \geqslant 0 .
\end{array}\right.
$$

By [15] there exists an exact solution $c$ for the deterministic fragmentation equation 

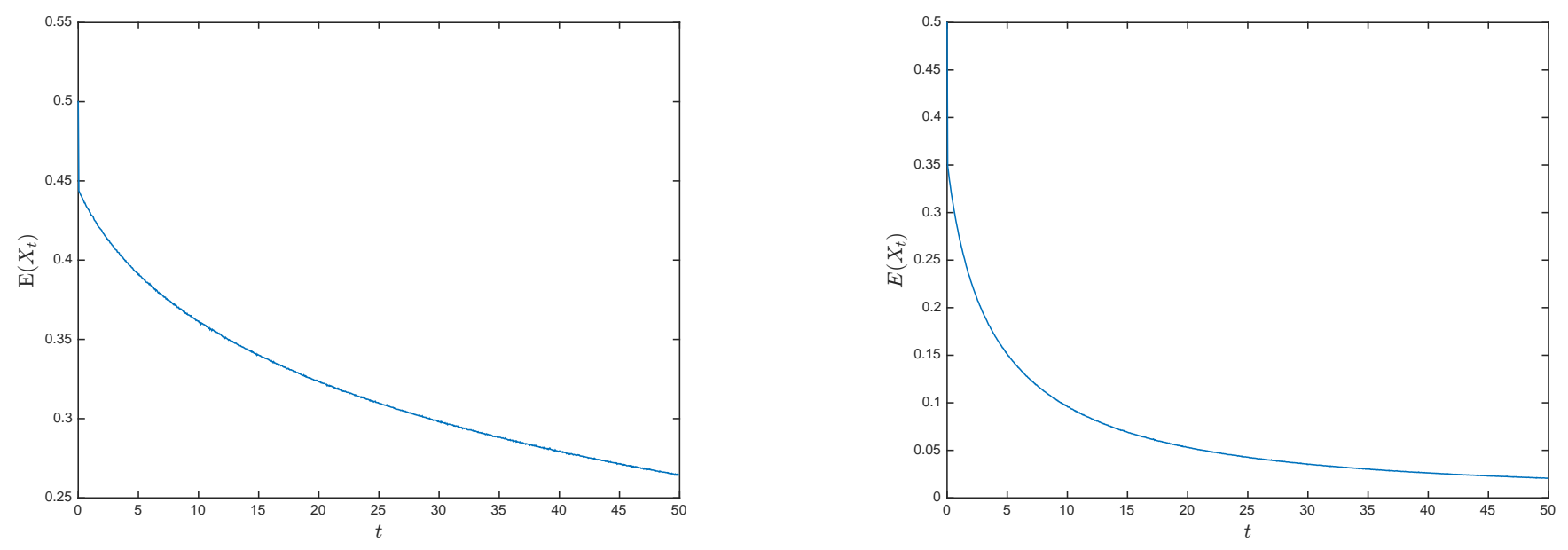

Figure 2: The path of $t \mapsto \hat{I}_{M}(t)$ for $F(x, y)=x+y$ (left) and $F(x, y)=2$ (right) for $t \in[0,50]$, the Monte Carlo parameter is $10^{4}$, and the Euler step $10^{-3}$.
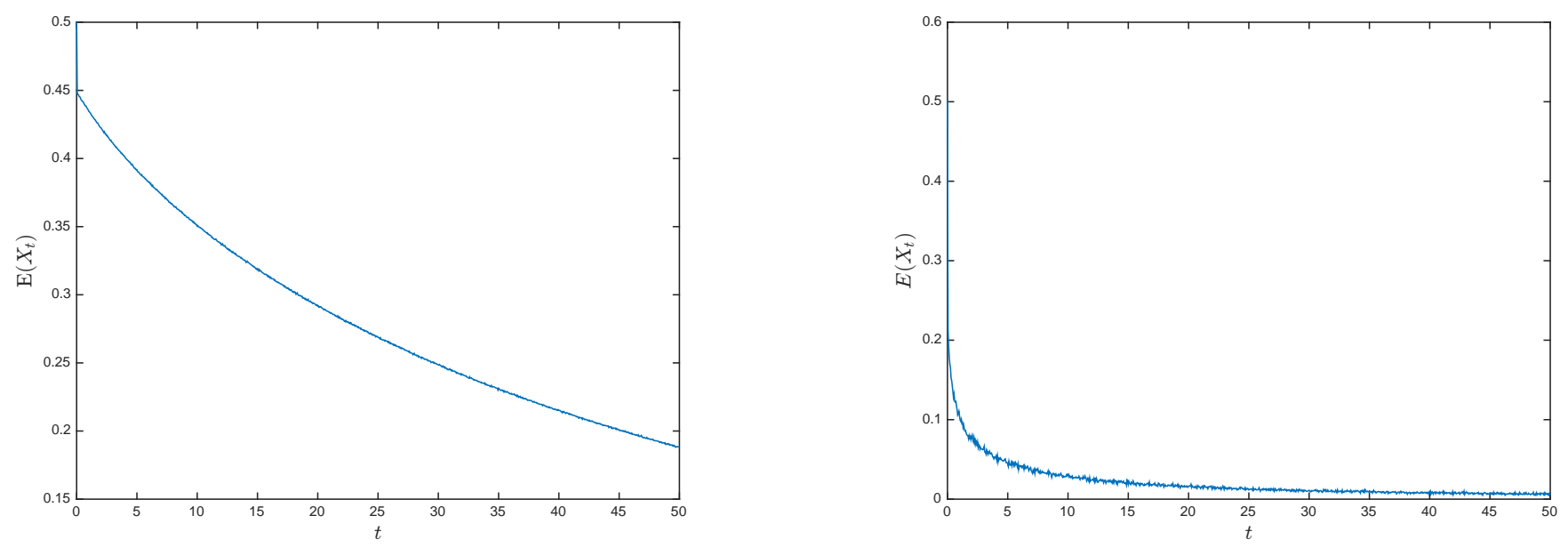

Figure 3: The path of $t \mapsto \hat{I}_{M}(t)$ for $F(x, y)=1 /(1+x+y)$ (left) and $F(x, y)=2 /(x+y)^{3}$ (right) for $t \in[0,50]$, the Monte Carlo parameter is $10^{4}$, and the time-step $10^{-3}$.

(4.1). Its form is

$$
c(t, x)=e^{-t x}\left(c(0, x)+\int_{x}^{\infty} c(0, y)\left(2 t+t^{2}(x-y)\right) \mathrm{d} y\right)
$$

and if we chose the initial condition $c(0, x)=e^{-x}$ we obtain this solution as

$$
c(t, x)=(1+t)^{2} e^{-x(1+t)} \text { for all } t \geqslant 0 \text { and } x \geqslant 0 .
$$


We compare this exact solution with the approximation of the stochastic process. We take the initial condition to be $Q_{0}(\mathrm{~d} x)=x e^{-x}$, if $x \geqslant 0$. To simulate such a density one can simply do the sum of two independent exponential random variables of parameter 1 .

In the Table 2 we compare for the fragmentation rate $F(x, y)=2$ the theoretical mean of the exact solution, which equals $\frac{2}{1+t}$, with the Monte Carlo mean, for different values of $t$ by using the associated stochastic process and the corresponding algorithm.

\begin{tabular}{|c|c|c|}
\hline$t$ & Mean $\widehat{I}_{M}$ & Exact solution \\
\hline 50 & 0.0415 & 0.0392 \\
\hline 70 & 0.0286 & 0.0282 \\
\hline 150 & 0.0126 & 0.0132 \\
\hline
\end{tabular}

Table 2: Monte Carlo estimators and exact solutions for $F(x, y)=2, Q_{0}(\mathrm{~d} x)=x e^{-x}$, $M=10^{6}$, and $T=50$.

\subsection{Fragmentation kernels for avalanches}

The first step in the numerical modeling of the fragmentation phase of an avalanche is based on the stochastic differential equation of fragmentation (2.10) with the discontinuous kernel $F^{r}$. We fix the parameter $\beta<\frac{1}{2}$ and a final time $T$. Using the numerical scheme stated in [5], Section 5, we have the following algorithm to obtain the paths of the process $\left(X_{t}\right)_{t \geq 0}$, solution of the equation (2.10).

\section{Algorithm}

Step 0: Sampling the initial particle $X_{0} \sim Q_{0}$

Step p: Sampling a random variable $S_{p} \sim \operatorname{Exp}\left(\lambda_{0}\right)$

$$
\begin{aligned}
& \text { Set } T_{p}=T_{p-1}+S_{p} \\
& \text { Set } X_{t}=X_{p-1} \text { for each } t \in\left[T_{p-1}, T_{p}\right) \\
& \text { Set } X_{p}=\beta X_{p-1} \text { with probability } \beta X_{p-1}, \\
& \quad X_{p}=(1-\beta) X_{p-1} \text { with probability }(1-\beta) X_{p-1} \text {, } \\
& \quad \text { or } X_{p}=X_{p-1} \text { with probability } 1-X_{p-1}
\end{aligned}
$$

Stop: When $T_{p}>T$.

Outcome: The approximated particle mass at time $T, X_{p-1}$.

In Figure 4 below we illustrate several paths of the processes $X_{T}$, using the above algorithm, corresponding to the fragmentation kernel related to the avalanches, as solution of the stochastic differential equation (2.10).

In Table 3 below we give the Monte Carlo estimator of the mean and the length of the confidence interval in the case of the discontinuous fragmentation kernel $F^{r}$.

The mean by Euler and Monte-Carlo. As in the case of a continuous fragmentation kernel, we sketch the evolution in time of $\mathbb{E}\left(X_{t}\right)$ for $t \in[0,50]$, see Figure 5 below. 

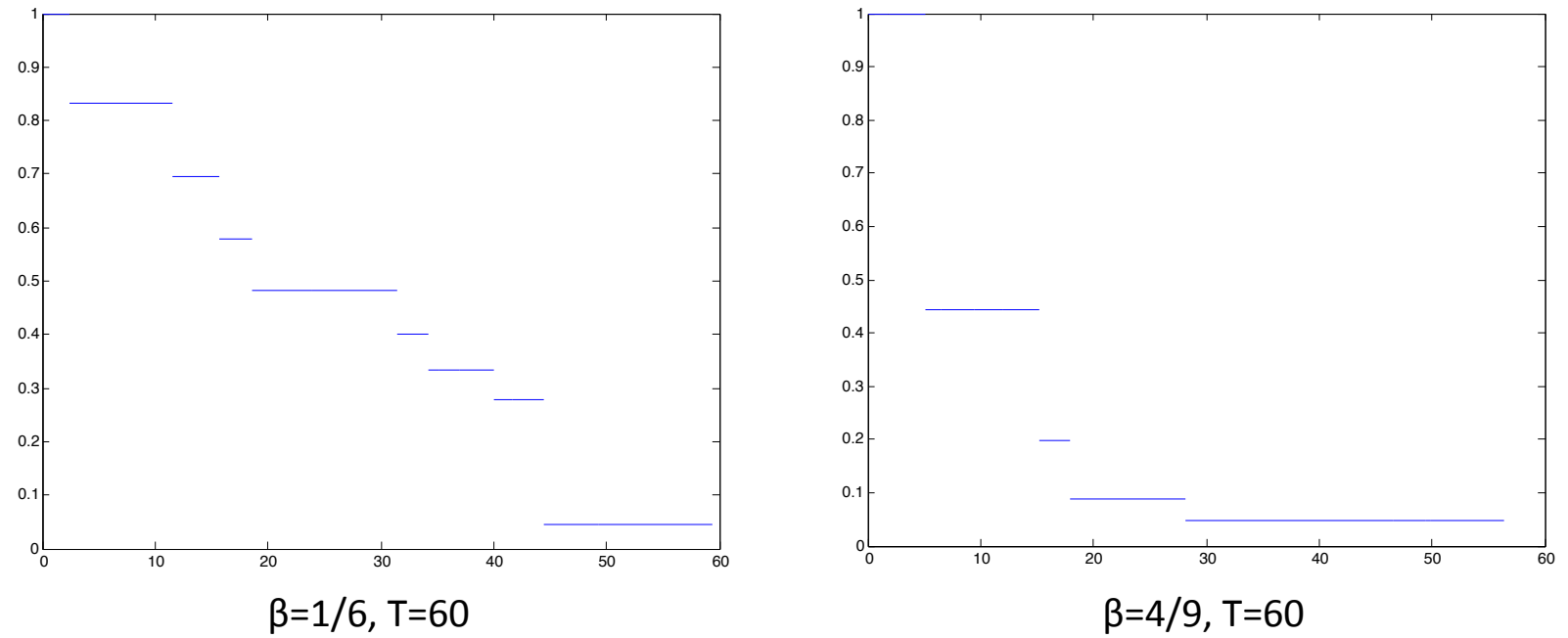

Figure 4: The paths of the fragmentation process with discontinuous kernel $F^{r}$ and the size of the initial particle 1 .

\begin{tabular}{|c|c|c|}
\hline$\beta$ & Mean $\widehat{I}_{M}$ & Confidence interval size (half) \\
\hline$\frac{1}{6}$ & 0.1566 & 0.0020 \\
\hline$\frac{1}{3}$ & 0.1356 & 0.0017 \\
\hline$\frac{4}{9}$ & 0.1350 & 0.0016 \\
\hline
\end{tabular}

Table 3: Monte Carlo estimators for $10^{4}$ simulations and $T=50$.

\subsection{The distribution of the branching process}

Considered on each interval $E_{n}$ the process $X^{n}$ from Propostion 2, having the transition function $\left(P_{t}^{n}\right)_{t \geqslant 0}$. It becomes the base process of the forthcoming branching process $\widehat{X^{n}}=\left(\widehat{X_{t}^{n}}\right)_{t \geqslant 0}$. We present now a numerical approach to compute the transition function $\left(\widehat{P_{t}^{n}}\right)_{t \geqslant 0}$ of the branching process $\widehat{X^{n}}$.

In Section 5 from [5] we gave an algorithm of approximating $h_{t}=\left.\widehat{P_{t}^{n}} \widehat{\varphi}\right|_{E_{n}}$ by Picard iterations $h_{t}^{k}, k \geqslant 0, t \leqslant T, \varphi \leq 1$. Below, in order to compute the distribution of the branching process, we complete this method, approximating also $P_{t}^{n} \varphi$ with iterations $p_{t}^{k}(\varphi), k \geqslant 0$.

In the sequel, to simplify the notations, we put $E=E_{n}, \widehat{X}=\widehat{X^{n}}, B^{n}=B, \mathcal{F}=\mathcal{F}_{n}^{r}$, and $P_{t}=P_{t}^{n}$.

- Initialization step: Set $h_{t}^{0}=e^{-t} P_{t} \varphi, p_{t}^{0}(\varphi)=\varphi$

- Step $k, k \geq 1$ :

$$
h_{t}^{k}=e^{-t} P_{t} \varphi+\int_{0}^{t} e^{-(t-u)} P_{t-u} B \widehat{h_{u}^{k-1}} \mathrm{~d} u, p_{t}^{k}(\varphi):=\sum_{i=0}^{k} \frac{t^{i}}{i !} \mathcal{F}^{i} \varphi
$$




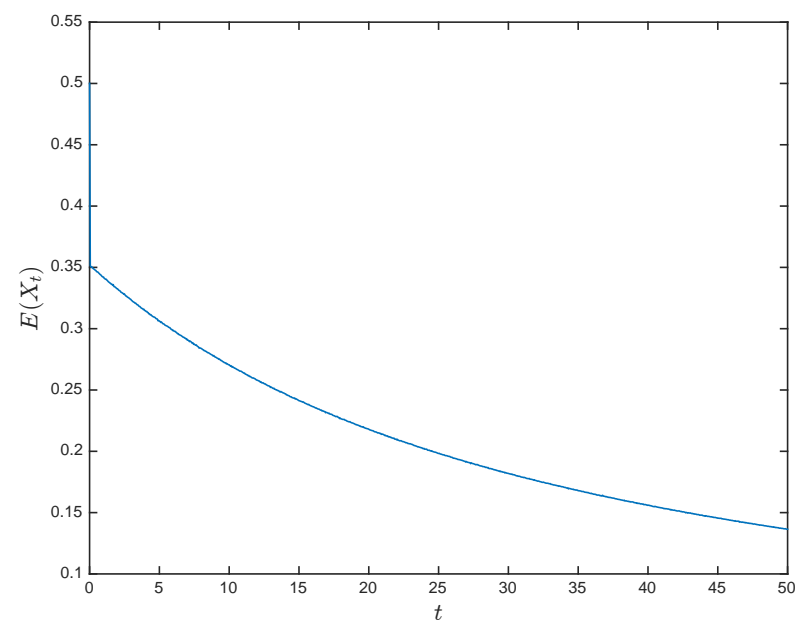

Figure 5: The path of $t \mapsto \hat{I}_{M}(t)$ for Monte Carlo parameter $M=10^{6}$, Euler step $10^{-3}$, and $t \in[0,50]$.

$$
h_{t}^{\prime k}:=e^{-t} p_{t}^{k}(\varphi)+\int_{0}^{t} e^{-(t-u)} p_{t-u}^{k-1}\left(B \widehat{h_{u}^{k-1}}\right) \mathrm{d} u .
$$

- Fix $\varepsilon>0$ and $m \geqslant 1$. By Remark $4.2(i v)$ from [5], which explains the connection between the transition functions of $X$ and of the branching process, we have $\left.\widehat{P}_{t} \widehat{\varphi}\right|_{E^{(m)}}=$ $\left.\widehat{h_{t}}\right|_{E^{(m)}}$. By Remark 4.2 (i) and (A3.2) from [6] and using (4.2) we can estimate the approximation errors:

$$
\begin{gathered}
\|\left(\left.\widehat{\left.P_{t} \widehat{\varphi}-\widehat{h_{t}^{\prime k}}\right)}\right|_{E^{(j)}} \|_{\infty} \leqslant m\left(\left\|h_{t}-h_{t}^{k}\right\|_{\infty}+\left\|h_{t}^{k}-h_{t}^{\prime k}\right\|_{\infty}\right) \leqslant\right. \\
e^{-t} m\left(\sum_{i \geqslant 0} e^{t} \frac{\left(2 t_{0}\right)^{k+i}}{(k+i) !}+\left\|P_{t} \varphi-p_{t}^{k}(\varphi)\right\|_{\infty}+\int_{0}^{t} e^{u}\left\|P_{t}\left(B \widehat{h_{u}^{k-1}}\right)-p_{t-u}^{k-1}\left(B \widehat{h_{u}^{k-1}}\right)\right\|_{\infty} \mathrm{d} u\right) \\
\left\|P_{t} \varphi-p_{t}^{k}(\varphi)\right\|_{\infty} \leqslant \sum_{i \geqslant 0} \frac{\left(\mu_{o} t_{0}\right)^{k+i}}{(k+i) !} \text { for all } j \leqslant m \text { and } t \leqslant t_{0},
\end{gathered}
$$

where $\mu_{o}:=\|\mathcal{F}\|$.

- Stop the algorithm at a convenient value of $k$ and $t_{0}$, such that

$$
\sum_{i \geqslant 0} \frac{\left(2 t_{0}\right)^{k+i-1}+\left(\mu_{o} t_{0}\right)^{k+i-1}}{(k+i-1) !}<\frac{\varepsilon}{2 m} .
$$

The following proposition shows that the above algorithm approximates indeed the distribution of the branching process. 
Proposition 7. Let $k$ be such that the inequality (4.5) holds, take $\varphi=1_{A}$ with $A \in \mathcal{B}(E)$, $\mathbf{x} \in E^{(j)}$, and $j \leqslant m$. Then $\widehat{h_{t}^{\prime k}}(\mathbf{x})$ approximates with error less than $\varepsilon$ the probability that the branching process $\widehat{X}$ starting from $\mathbf{x}$ lies at the time moment $t$ in the set $A^{j}$, i.e.,

$$
\left|\mathbb{E}^{\mathbf{x}}\left(\widehat{X}_{t} \in A^{j}\right)-\widehat{h_{t}^{\prime k}}(\mathbf{x})\right|<\varepsilon .
$$

Proof. The assertion follows from (4.3), (4.4), and (4.5).

Numerical results. In order to give an approximation value for the distribution of the branching process $\widehat{X^{n}}$, we implement the numerical scheme introduced above as follows. We take: $m=2, n=1$ and so $E=E_{1}=\left[\frac{1}{4}, 1\right], \beta=\frac{4}{9}$ and thus $\mu_{o}=2 \lambda_{o}=\frac{6}{25}$. Let further $\mathbf{x}=(1,1, \ldots)$.

Consider $A:=E_{\frac{4}{9}, 1} \cap E_{1}=\left\{\left(\frac{5}{9}\right)^{2}, \frac{4}{9}, \frac{5}{9}, 1\right\}$. By assertion (iv) of Theorem 3 we know that $\mathbb{E}^{\mathbf{x}}\left(\widehat{X}_{t} \in \widehat{A}\right)=\widehat{P}_{t}\left(1_{\widehat{A}}\right)(\mathbf{x})=1$.

On the other hand, observing that $\mathcal{F} \varphi(1)=\frac{3}{25}\left(\frac{4}{9} \varphi\left(\frac{4}{9}\right)+\frac{5}{9} \varphi\left(\frac{5}{9}\right)-\varphi(1)\right)$, using the algorithm for $k=1$ in the Picard method (4.2), taking $\varepsilon=0.5$ and $t_{0}=0.05$, one can see that (4.5) holds and we have $h_{t_{0}}^{\prime 1}=0.9998$. According to Proposition 7 , we conclude by this computation that the approximate value of $\mathbb{E}^{\mathbf{x}}\left(\widehat{X}_{t_{0}} \in \widehat{A}\right)$ (= the probability that the branching process $\widehat{X}$ starting from $\mathbf{x}$ lies in the set $\widehat{A}$ at the time moment $\left.t_{0}\right)$ is $\widehat{h_{t_{0}}^{\prime 1}}(\mathbf{x})=0.9996$, which is indeed a value from the error interval $(1-\varepsilon, 1]$.

We finally note that the above Picard iterations have been recently used in [14] to give a probabilistic numerical approach for a nonlinear Dirichlet problem associated with a branching process.

\section{Summary}

We investigate real-life phenomena of rupture-type, using probabilistic methods, having as application a probabilistic model for the fragmentation phase of an avalanche. First, we present the theoretical model, based on branching-fragmentation processes and specific stochastic differential equations. The efficiency of this approach is emphasized by the numerical results, obtained with our simulation methods in Section 4, results which are close to the theoretical ones.

\section{References}

[1] Ch. Ancey, Plasticity and geophysical flows: A review, J. Non-Newtonian Fluid Mech., 142 (2007), 4-35.

[2] J. Bertoin, Random Fragmentation and Coagulation Processes, Cambridge Univ. Press, 2006.

[3] L. Beznea and I. Cîmpean, On Bochner-Kolmogorov theorem, in Séminaire de Probabilités XLVI (LNM, vol. 2123), Springer, 2014, pp. 61-70.

[4] L. Beznea, M. Deaconu and O. Lupaşcu, Branching processes for the fragmentation equation, Stoch. Proc. Appl., 125 (2015), 1861-1885. 
[5] L. Beznea, M. Deaconu and O. Lupaşcu, Stochastic equation of fragmentation and branching processes related to avalanches, J. Stat. Phys., 162 (2016), 824-841.

[6] L. Beznea and O. Lupaşcu, Measure-valued discrete branching Markov processes, Trans. Amer. Math. Soc., 368 (2016), 5153-5176.

[7] L. Beznea and A.-G. Oprina, Nonlinear PDEs and measure-valued branching type processes, J. Math. Anal. Appl., 384 (2011), 16-32.

[8] R.M. Blumenthal and R.K. Getoor, Markov Processes and Potential Theory, Academic Press, 1968.

[9] X. Bressaud and N. Fournier, On the invariant distribution of a one-dimensional avalanche process, Ann. Probab., 37 (2009), 48-77.

[10] N. Fournier and J.-S. Giet, On small particles in coagulation-fragmentation equations. J. Stat. Phys., 111 (2003), 1299-1329.

[11] M. Deaconu, N. Fournier and E. Tanre, Rate of convergence of a stochastic particle system for the Smoluchowski coagulation equation, Methodol. Comput. Appl. Probab., 5 (2003), 131-158.

[12] N. Ikeda, M. Nagasawa and S. Watanabe, Branching Markov processes I, J. Math. Kyoto Univ., 8 (1968), 233-278.

[13] J. Jacod, Calcul Stochastique et Problèmes de martingales (LNM, vol. 714), Springer, 1979.

[14] O. Lupaşcu and V. Stănciulescu, Numerical solution for the non-linear Dirichlet problem of a branching process, Compl. Anal. Oper. Th., (2017), DOI: 10.1007/s11785017-0642-z.

[15] E.D. McGrady and R. M. Ziff, Analytical solutions to fragmentation equations with flow, AIChE J., 34 (1988), 2073-2076.

[16] S. Shindin and N. Parumasur, Numerical simulation of a transport fragmentation coagulation model, Appl. Math. Comput., 246 (2014), 192-198.

[17] M.L. Silverstein, Markov processes with creation of particles, Z. Warsch. verw. Geb., 9 (1968), 235-257.

[18] S. P. Pudasaini and K. Hutter, Avalanche Dynamics - Dynamics of Rapid Flows of Dense Granular Avalanches, Springer, 2007.

[19] L.E. Vallejo, S. Lobo-Guerrero and Z. Chik, A Network of Fractal Force Chains and Their Effect in Granular Materials under Compression, in Fractals in Engineering, Springer 2005, pp. 67-80. 